\title{
Elevated body mass in National Football League players linked to cognitive impairment and decreased prefrontal cortex and temporal pole activity
}

\author{
K Willeumier, DV Taylor and DG Amen
}

Obesity is a risk factor for neurodegenerative disease and has been shown to adversely affect cognitive function. Professional athletes who participate in sports, which expose them to repetitive concussions, may be at heightened risk for cognitive impairment. Here, we investigated the effects of body mass as measured by waist-to-height ratio (WHtR) on regional cerebral blood flow using single-photon emission computed tomography imaging in 38 healthy weight (WHtR mean $49.34 \pm 2.8$; age $58 \pm 9.6$ ) and 38 overweight (WHtR mean $58.7 \pm 4.7$; age $58 \pm 13.3$ ) retired National Football League football players. After matching for age and position, we used a two sample $t$-test to determine the differences in blood flow in healthy versus overweight subjects. Statistical parametric mapping revealed a higher WHtR ratio is associated with decreased blood flow in Brodmann areas 8, 9 and 10, brain regions involved in attention, reasoning and executive function $(P<0.05$, family-wise error) along with deficits in the temporal pole. Moreover, overweight athletes had significant decrease in attention $(P=0.01326)$, general cognitive proficiency $(P=0.012$; Microcog: Assessment of Cognitive Functioning) and memory $(P=0.005$; Mild Cognitive Impairment Screen). The association between elevated WHtR percentage and decreased blood flow in the prefrontal cortex and temporal pole may be correlated with the decreased performance on tests of attention and memory. These findings suggest that a weight management program may be critical to the health of athletes who have been exposed to mild brain trauma during their careers.

Translational Psychiatry (2012) 2, e68; doi:10.1038/tp.2011.67; published online 17 January 2012

\section{Introduction}

Obesity is a growing epidemic in youth, ${ }^{1}$ collegiate $^{2}$ and professional football players, ${ }^{3}$ thus, potentially escalating the risk for cognitive impairment in a group that is already at risk due to repetitive subconcussive and concussive brain trauma. Recent studies have highlighted the impairments in executive cognitive function in overweight healthy adults, ${ }^{4}$ but no study to date has investigated the effects of weight on brain function in professional athletes. This is a unique population of strength-trained individuals who are required to have a higher body mass for competitive purposes. Body mass index was found not to be a valid indicator of overweight and obesity in a collegiate football population, ${ }^{5}$ suggesting that the waist-toheight ratio $(\mathrm{WHtR})$ is a more appropriate assessment of the healthy weight of an amateur or professional athlete. We have observed that retired football players are susceptible to weight gain. ${ }^{6}$ Therefore, it is important to evaluate if the combination of weight gain and brain trauma in football players has an additive negative effect on brain function.

Using single-photon emission computed tomography (SPECT) imaging, we have previously demonstrated significant global decreases in blood flow in a study of 100 retired professional National Football League (NFL) football players. ${ }^{7}$ In addition, we recently found a decrease in blood flow in the prefrontal cortex of normal, healthy individuals who have an elevated body mass. ${ }^{8}$ On the basis of our previous findings, we hypothesized that similar blood flow deficits and cognitive deficits are present in a group of overweight athletes who have been exposed to repetitive subconcussive or concussive collisions as compared with normal-weight players.

To test our hypothesis, we measured regional cerebral blood flow (rCBF) in 38 normal-weight and 38 overweight retired NFL players who had previously visited our clinic to participate in a brain trauma study. We utilized SPECT imaging as a measure of blood flow, and we administered the Microcog: Assessment of Cognitive Functioning (MACF) and Mild Cognitive Impairment Screen (MCIS) to assess mental performance and attention. We hypothesized an inverse correlation between $\mathrm{WHtR}$ percentage and scores on attention, reasoning and general cognitive function in addition to significant deficits in blood flow in the prefrontal cortex.

\section{Materials and methods}

Subjects. We recruited a total of 100 active and retired players, representing 27 teams and all positions to participate in a study measuring the impact of playing professional American football on long-term brain function ${ }^{7}$ 
Table 1 Demographics

\begin{tabular}{lc}
\hline Mean Age & $57.27+12.369,($ range 25-82) \\
Handedness & 84 right, 16 left or ambidextrous \\
Ethnicity & \\
African American & 33 \\
Caucasian & 60 \\
Hispanic & 1 \\
Mixed & 6 \\
& \\
Positions (N=100) & \\
Quarterback & 5 \\
Running back & 8 \\
Wide Receivers & 7 \\
Tight Ends & 7 \\
Offensive lineman & 26 \\
Defensive lineman & 12 \\
Linebackers & 17 \\
Defensive backs & 18 \\
\hline
\end{tabular}

(see Table 1 for demographics). Players were recruited from retired NFL Players Association meetings and by participants informing other players about the study. Of these 100 subjects, 76 were participants in this study, as we were able to attain their waist sizes. Each player met our inclusion criteria of being on an active NFL roster for a minimum of 3 years. We excluded subjects who could not cease taking psychoactive medications (recreational or otherwise) for an appropriate washout period before scanning. In all, $12 \%$ of the participants had a prior or current history of psychoactive medication use. All subjects received an explanation of the research study and gave written informed consent in accordance with an Institutional Review Board-approved protocol.

Evaluation. Each participant was interviewed by a physician and completed a detailed history. Weight and height were obtained on all participants and a WHtR was calculated by dividing the waist circumference in inches by height in inches.

Neuropsychological exams. As part of the evaluation the MACF was administered. This exam measures the following nine parameters: general cognitive functioning, general cognitive proficiency, information processing speed, information processing accuracy, attention, reasoning, memory, spatial processing and reaction time. The MACF scores were compared with its own standardized sample ( $n=810$ ) chosen to be representative to the US population of adults with regard to education, gender and ethnicity between the ages of 18 and 89. Participants were also given the $\mathrm{MCIS},{ }^{9}$ a screening tool found to be reliable in distinguishing mild cognitive impairment from normal. ${ }^{10}$

SPECT scans. In addition, each subject underwent highresolution brain SPECT imaging to measure rCBF. Each subject received an age/weight-appropriate dose of Technetium-99m hexamethylpropyleneamine oxime (Tc99m HMPAO) intravenously. Subjects were injected in normal lighting while they performed the Conner's Continuous Performance Test II. The radiopharmaceutical was injected $3 \mathrm{~min}$ after starting the 15-min test. All subjects completed the task. Subjects were then scanned 30 min later using a high-resolution Picker Prism 3000 triple-headed gamma camera (Picker Int. Inc., Ohio Nuclear Medicine Division, Bedford Hills, OH, USA) with fan beam collimators, acquiring data in $128 \times 128$ matrices, yielding 120 images per scan with each image separated by $3^{\circ}$ spanning $360^{\circ}$.

SPECT data were processed and attenuation correction performed using general linear (Chang) methods. All images were reconstructed and resliced using an oblique reformatting program, according to anterior-posterior commissure line so that the final images were similarly aligned for analysis.

Image analysis. Differences in HMPAO uptake were analyzed using Statistical Parametric Mapping 8 software (Wellcome Department of Cognitive Neurology, London, UK) implemented on the Matlab platform (Math Works, Sherborn, MA, USA). Statistical parametric maps (SPMs) are spatially extended statistical processes that are constructed to test hypotheses about regionally specific effects in neuroimaging data. SPM combines the general linear model and the theory of Gaussian random fields to make statistical inferences about regional effects. ${ }^{11}$ The images were spatially normalized using a 12-parameter affine transformation followed by non-linear deformations ${ }^{12}$ to minimize the residual sum of squares between each scan and a reference or template image conforming to the standard space defined by the Montreal Neurological Institute template. The original image matrix obtained at $128 \times 128 \times 29$ with voxel sizes of $2.16 \times 2.16 \times 6.48 \mathrm{~mm}$ were transformed and resliced to a $79 \times 95 \times 68$ matrix with voxel sizes of $2 \times 2 \times 2 \mathrm{~mm}$ consistent with the Montreal Neurological Institute template. The normalized images were smoothed using an 8-mm full-width half maximum isotropic Gaussian kernel.

Statistical analysis. SPECT scans from age- and positionmatched normal-weight players were compared with overweight retired NFL players using statistical parametric mapping (SPM) with t-contrast modeling. The linear relationship between $\mathrm{WHtR}$ and blood flow was evaluated using the SPM multiple regression model. The differences in general cognitive function, general cognitive proficiency, reasoning, attention and memory were evaluated using SPSS statistical software for Windows (SPSS, Chicago, IL, USA) and $P<0.05$ was considered statistically significant.

\section{Results}

Elevated WHtR decreases cognitive performance and rCBF. Average WHtR in our overweight group was $58.7 \pm 4.7$ (range 53-72.97) and in our healthy weight group was $49.34 \pm 2.8$ (range 41.03-52.9) with the means being statistically different $\left(t_{(2,74)}=10.473, \quad P<0.001\right)$. Those with a WHtR percentage under 53\% were categorized as healthy weight. We matched players by age and position based on findings from our previous work with this NFL group which demonstrated a significant relationship between position and cognitive function. ${ }^{7}$ 
Initially, we examined results of the general cognitive proficiency and the general cognitive function as an assessment of cognition in those with increased WHtR using the MACF. A between-group ANOVA demonstrated decreases in general cognitive proficiency $(P=0.017)$ and general cognitive function $(P=0.025)$ as a main effect. The decrease in general cognitive proficiency remained significant after Bonferroni correction for the two comparisons $(P<0.025)$. To discern which factors contributed to decreased general cognitive proficiency, we evaluated attention, memory, reasoning, information processing speed, and information processing accuracy between the two groups. These variables were previously demonstrated to be areas of deficit as well as sensitive to improvement in our previous study. ${ }^{6}$ Consistent with the decrease in general cognitive proficiency, information processing accuracy was significantly decreased $(P=0.024)$ but not information processing speed; general cognitive proficiency is more sensitive to processing accuracy than speed. Of the three underlying domains that contribute to an overall decrease in cognitive proficiency, we found decreases in reasoning $(P=0.023)$ and attention $(P=0.023)$ but not memory in those with elevated WHtR using the MCAF. An independent evaluation of memory function using the $\mathrm{MCIS}$, which is sensitive to age-related memory impairment, revealed significant deficits with increased body mass $(P=0.003)$

Our initial SPM analysis on brain SPECT concentration scans revealed global diffuse decreases in blood flow in the frontal, temporal and parietal lobes of subjects in the elevated WHtR group at $P<0.001$, uncorrected for family-wise error (FWE; Figure 1). We then refined our analysis to include regions associated with focus, impulsivity and attention (Brodmann areas 8, 9, 10, 11 and 44 ) based on previous data demonstrating elevated body mass index results in decreased executive function ${ }^{4,13}$ metabolic activity ${ }^{14}$ and blood flow ${ }^{8}$ in the prefrontal cortex. This analysis emphasized the regions of significant decreases were specific to Brodmann areas 8, 9 and 10 in the elevated WHtR group at $P<0.05$ FWE. Figure 2 illustrates the three-dimensional cortical representation of these brain regions and Table 2 provides the regional areas of significant decrease with the largest cluster size as identified through SPM and defined by the Automated Anatomical Labeling Atlas. These areas include the left and right superior and middle frontal gyrus. No significant increases were observed.

As body mass increases frontal and temporal lobe perfusion decreases. Linear regression analysis at $P<0.001$ FWE measuring the effect of WHtR on blood flow while controlling for age, predicts the most significant decreases as reported by voxel cluster size in the following five regions: Region 1 has an overall cluster size of 2666 voxels and includes the left superior temporal pole (coordinates $-40,16,-20 ; t=6.91$ ), left frontal medial orbital cortex (coordinates $-2,30,-14 ; t=6.79$ ) and left insular cortex (coordinates $-28,18,-16$; and $t=6.78$ ). Region 2 has an overall cluster size of 207 voxels and includes the left frontal medial orbital cortex (coordinates -8 , $56,-14 ; t=6.35$ ) and right rectus (coordinates $8,56,-18$; $t=6.35$ ). Region 3 has an overall cluster size of 342 voxels

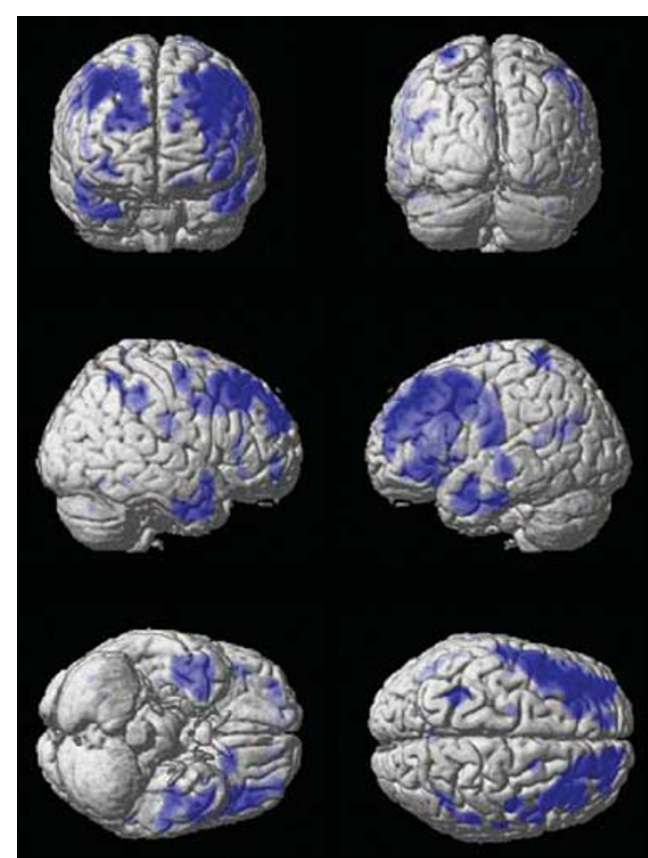

Figure 1 Three-dimensional surface images showing global regions of decreased perfusion in the elevated WHtR cohort at $P<0.001$ (uncorrected). Cortical surface views showing significant hypoperfusion in NFL players with elevated WHtR $(n=38)$ compared with those who have a normal WHtR $(n=38)$. Regions showing the most hypoperfusion include the bilateral frontal, temporal and parietal lobes, and cerebellum. The statistical threshold was set at $P<0.001$. Significant decreases are in blue. No increases were observed.

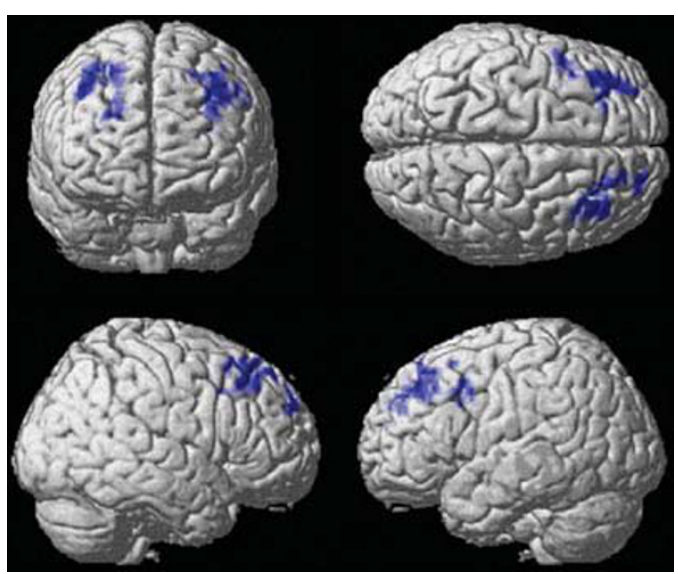

Figure 2 Three-dimensional surface images showing areas of decreased perfusion in the prefrontal cortex with elevated WHtR at $P<0.05$. Cortical surface views demonstrate decreased regional cerebral blood flow in Brodmann areas 8, 9, 10 in players with a high WHtR at $P<0.05$, family-wise error (FWE). Significant decreases are in blue. No increases were observed.

and includes the right superior temporal pole (coordinates $48,10,-14 ; t=6.23$ and coordinates $32,12,-32 ; t=7.10$ ). Region 4 has an overall cluster size of 253 voxels at the left middle frontal cortex (coordinates -32, 54, 20; $t=6.22$ ). Region 5 has an overall cluster size of 90 voxels and includes the frontal superior right cortex (coordinates 14, $-42,52$; $t=6.17$ and coordinates $18,54,42 ; t=5.96)$. Figure 3 
illustrates these decreased areas of perfusion through transverse, coronal and sagittal slices. Furthermore, a plot of the regression slope of region 1 (left superior temporal pole and left frontal medial orbital cortex) demonstrates a negative correlation between $\mathrm{WHtR}$ and blood flow at $P<0.001$ FWE (Figures 4 and 5). This suggests that as body mass increases, the blood flow to both temporal pole and prefrontal cortex decreases.

\section{Discussion}

Obesity is a worldwide epidemic and is becoming an issue of concern in certain contact sports like football. ${ }^{1,3,5,15}$ Previously, we have demonstrated significant global decreases in blood flow in a population of retired NFL players compared

Table 2 Result of SMP analysis on PFC regions (BA 8, 9, 10, 11, 44) showing areas of decreased $\mathrm{rCBF}$ in players with elevated compared with low $\mathrm{WH} \mathrm{HR}$ at $P<0.05$ FWE

\begin{tabular}{lccc}
\hline BA & $\begin{array}{c}\text { Cluster } \\
\text { size }\end{array}$ & $\begin{array}{c}\text { MNI coordinates } \\
(\boldsymbol{x}, \boldsymbol{y}, \boldsymbol{z})\end{array}$ & $\begin{array}{c}\text { Z-value at local } \\
\text { maximum }\end{array}$ \\
\hline BA 8 & 297 & $36,30,50$ & 5.85 \\
BA 9 & 280 & $-38,36,40$ & 5.19 \\
BA 8 & 105 & $-46,10,42$ & 4.83 \\
BA 10 & 46 & $24,56,30$ & 4.22
\end{tabular}

Abbreviations: BA, Brodmann area; FWE, family-wise error; MNI, Montreal Neurological Institute; PFC, prefrontal cortex; rCBF, regional cerebral blood flow; SMP, statistical parametric mapping; WHtR, waist-to-height ratio. with healthy subjects, ${ }^{7}$ and we have observed decreases in blood flow to the prefrontal cortex in a group of healthy, cognitively normal individuals who were overweight. ${ }^{8}$ Here, we show that retired, overweight NFL players have significantly more decreases in blood flow in the prefrontal cortex and temporal pole, and greater cognitive impairment than those retired NFL players categorized as normal weight. It appears that both playing professional football and being

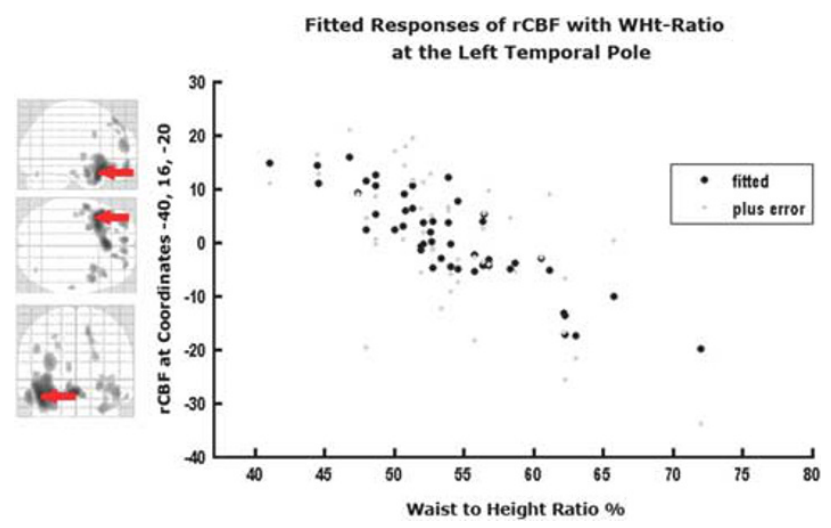

Figure 4 Left temporal pole hypoperfusion correlates with elevated WHtR at $P<0.001$ FWE. Negative correlation between $\mathrm{rCBF}$ in the left temporal pole and WHtR percentage in NFL players. Images represent regions of the brain that are significantly decreased with elevated WHtR $(P<0.001$, family-wise error). The arrow represents the center of the cluster in the regression model.

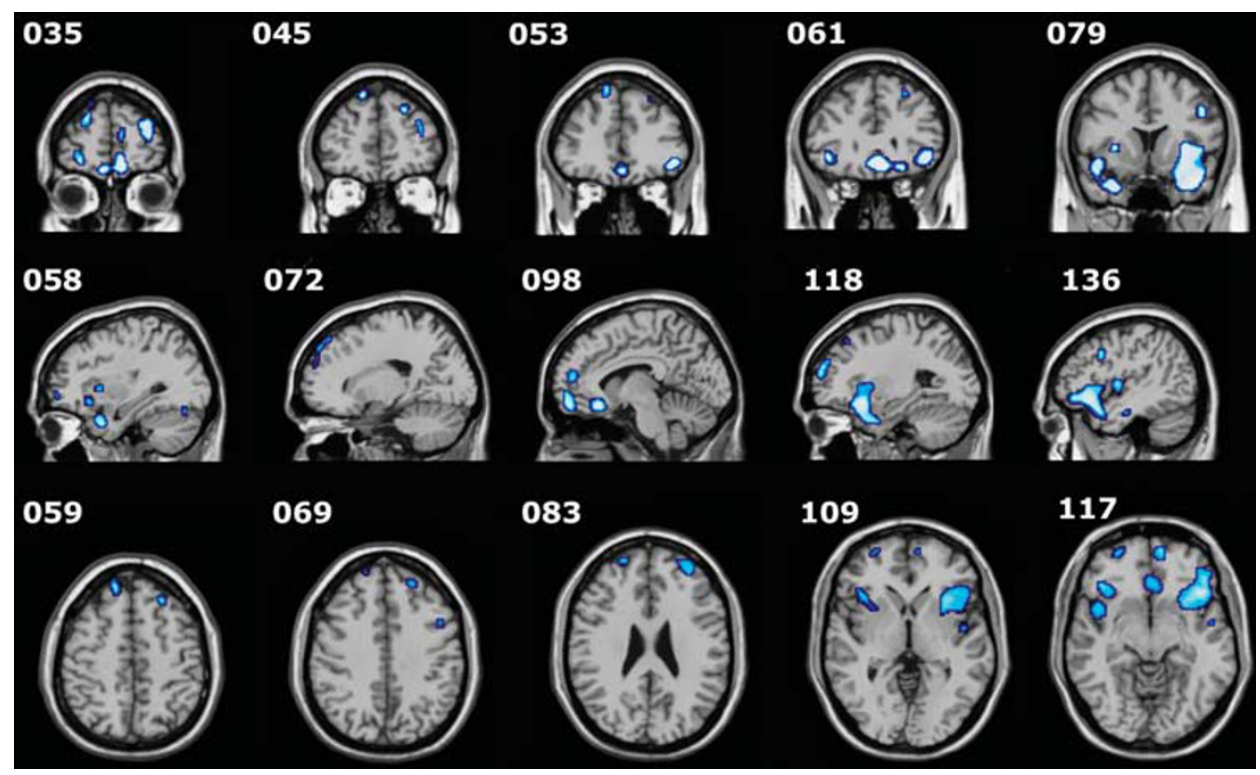

Figure 3 Image sections showing regional cerebral blood flow (rCBF) decreases with elevated WHtR. Following a regression analysis of $\mathrm{rCBF}$ to WHtR, slices from coronal, sagittal and transverse sections show areas of significant hypometabolism: coronal, 035: right superior frontal cortex; right superior orbital frontal cortex; left middle frontal cortex; left superior medial frontal cortex; 045: right superior frontal cortex; left superior frontal cortex; left middle frontal cortex; 053: right superior frontal cortex; left precentral; left temporal pole; left medial inferior orbital frontal cortex; 061: right inferior orbital frontal cortex; left precentral; left temporal pole; left insula; left medial orbital frontal cortex; 079: left rolandic operculum ;left inferior temporal; right superior temporal pole. Sagittal, 058: right frontal inferior orbit; right putamen ; right superior temporal pole; right insula; 072: right mid frontal cortex right superior frontal cortex; 098: left medial orbital frontal cortex; left superior medial frontal cortex ;left medial orbital frontal cortex; 118: left mid frontal cortex; left superior frontal cortex; left temporal pole; 136: left precentral; left rolandic operculum ; left inferior temporal cortex; left superior temporal pole. Transverse; 059: right frontal superior cortex; left mid frontal cortex; 069: left superior frontal cortex; left rolandic operculum; 083: right mid frontal cortex; left mid frontal cortex; 109: right superior orbital cortex; left medial orbital frontal cortex; left temporal pole; left rolandic operculum; 117: right superior orbital frontal cortex, left medial orbital frontal cortex; left temporal pole, right superior temporal pole. 


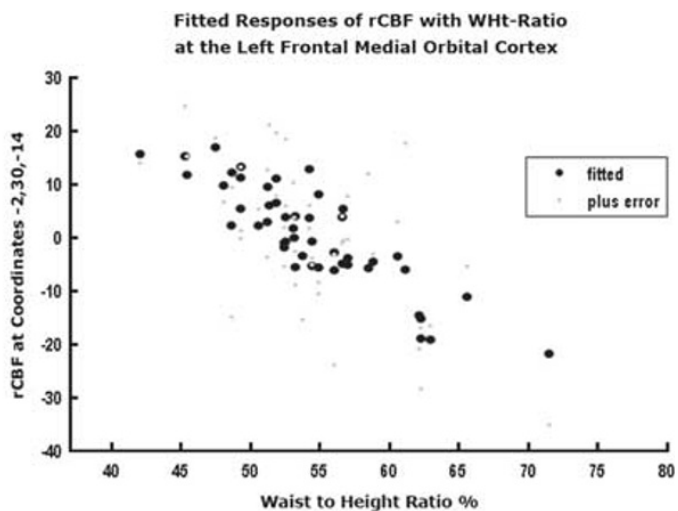

Figure 5 Medial orbital frontal hypoperfusion correlates with elevated WHtR at $P<0.001$ FWE. Negative correlation between rCBF in the medial orbital frontal cortex and WHtR percentage in NFL players. Images represent regions of the brain that are significantly decreased with elevated WHtR $(P<0.001$, family-wise error). The arrow represents the center of the cluster in the regression model.

overweight result in additive risk factors for decreasing blood flow and cognition.

The prefrontal cortex is the brain's executive processing center, allowing for cognitive flexibility, planning and forethought. This circuitry is involved in gathering and consolidating input required for complex behavior, thus allowing for a top-down processing of information. ${ }^{16,17}$ In essence, the prefrontal cortex functions as a highly tuned processing center with projections to the sensory and motor cortical systems, ultimately allowing for the output of proper goal-oriented behavior. When this connectivity becomes disrupted, deficits in cognitive flexibility, information processing speed, reasoning and attention ultimately result. In our cohort of overweight NFL players, we observed significant blood flow deficits specifically in the dorsolateral prefrontal cortex (Brodmann areas 8,9$)$, regions involved with processing of visuospatial and motor information and the anterior prefrontal cortex (BA $10)$, a region involved in memory retrieval and executive function. We also observed significant hypometabolism in the left temporal pole, which is associated with a variety of higherorder cognitive processes including emotion, attention, behavior and memory. As the temporal pole has extensive connectivity with the hippocampus and the frontal lobes, the blood flow deficits observed in the overweight players correlate well with the cognitive processing impairments present on the neuropsychiatric examinations.

Mild traumatic brain injury results from repetitive subconcussive impacts to the head and football players at the high school, collegiate and professional level are vulnerable to the cognitive deficits and long-term neurodegenerative complications that may accompany it. It is without question that NFL players are at a heightened risk for cognitive impairment ${ }^{18}$ and dementia. Although the pathogenesis underlying traumatic brain injury is not clearly understood, histological data demonstrate that the deposition of tau proteins, amyloid plaques and neurofibrillary tangles following repetitive head injury can increase susceptibility to chronic traumatic encephalopathy, a progressive neurological disorder documented in the autopsied brains of professional athletes involved in contact sports. ${ }^{19-21}$ The mechanical forces from repetitive head trauma rupture delicate cell membranes and alter the fidelity of the electrical signal that has been observed by our group ${ }^{7}$ and others. ${ }^{22}$ These subtle neurobiological changes that can influence cognition may ultimately usher in the more chronic effects of traumatic brain injury, including diffuse axonal injury, ischemia, synaptic loss and demyelination. The combined metabolic changes and alterations in neuronal integrity result in the global hypometabolism that we have previously reported in this population of athletes. ${ }^{7}$

In addition to the obvious risk factors from playing contact sports, the additive impact of having excess body mass increases the propensity for further metabolic and neurological complications. Anstey et al. $^{23}$ reported the results of a comprehensive meta-analysis on the association between body mass index and dementia, and found that categorization in the overweight or obese range in midlife is a risk factor for dementia later in life. Obesity results in an upregulation of proinflammatory cytokines (C-reactive protein, tumor necrosis factor $\alpha$ or interleukin-6) with a corresponding decrease in beneficial adipokines, triggering a cascade of apoptosis, neuroinflammation, microglia activation and blood-brain barrier damage often observed with metabolic syndrome. Brain imaging studies have reported the deleterious effects of having a high body mass index being associated with lower brain volume ${ }^{24}$ and impaired frontal lobe function. ${ }^{4,8,25}$ Therefore, it is clear that obesity has a role in compromised brain function, and we report here that retired NFL players who are overweight have greater cognitive impairment and blood flow deficits in the prefrontal cortex and temporal pole as compared with their normal-weight cohorts.

Obesity has been linked to executive dysfunction, ${ }^{4,13,25}$ and our cohort of overweight NFL players demonstrate decreased performance in the areas of attention and cognitive functioning upon neuropsychological evaluation. These observations may correlate with the decrements in metabolic activity to the prefrontal cortex and temporal pole that we observe in our overweight athletes. Alternatively, the cognitive deficits may be attributed to the elevated circulation of pro-inflammatory cytokines and biomarkers that result in myelin abnormalities, which have been documented in the frontal lobes of obese individuals. ${ }^{26}$ With the heightened prevalence of obesity $^{3}$ and cardiovascular disease risk factors ${ }^{27}$ in NFL players, our results suggest this trend will have negative, longterm consequences on brain health and may lead to complications due to stoke or chronic traumatic encephalopathy. If this finding is replicated it indicates that proper weight education and management may be essential to the future health of athletes who have been exposed to repetitive brain trauma.

\section{Conflict of interest}

The authors declare no conflict of interest.

Author contributions: KW performed recruitment, testing and project management, and manuscript preparation. DT did the data analysis. DGA is the primary investigator and evaluator of subjects.

1. Malina RM, Morano PJ, Barron M, Miller SJ, Cumming SP, Kontos AP et al. Overweight and obesity among youth participants in American football. J Pediatr 2007; 151: 378-382. 
2. Kaiser GE, Womack JW, Green JS, Pollard B, Miller GS, Crouse SF. Morphological profiles for first-year National Collegiate Athletic Association Division I football players. J Strength Cond Res 2008; 22: 243-249.

3. Harp JB, Hecht L. Obesity in the National Football League. JAMA 2005; 293: 1061-1062.

4. Gunstad J, Paul RH, Cohen RA, Tate DF, Spitznagel MB, Gordon E. Elevated body mass index is associated with executive dysfunction in otherwise healthy adults. Compr Psychiatry 2007; 48: 57-61.

5. Mathews EM, Wagner DR. Prevalence of overweight and obesity in collegiate American football players, by position. J Am Coll Health 2008; 57: 33-38.

6. Amen DG, Wu JC, Taylor D, Willeumier K. Reversing brain damage in former NFL players: implications for traumatic brain injury and substance abuse rehabilitation. J Psychoactive Drugs 2011; 43: 1-5.

7. Amen DG, Newberg A, Thatcher R, Jin Y, Wu J, Keator D et al. Impact of playing American professional football on long-term brain function. J Neuropsychiatry Clin Neurosci2011; 23 98-106.

8. Willeumier KC, Taylor DV, Amen DG. Elevated BMI is associated with decreased blood flow in the prefrontal cortex using SPECT imaging in healthy adults. Obesity (Silver Spring) 2011; 19: 1095-1097.

9. Medical Care Corporation. Mild Cognitive Impairment Screen. Medical Care Corporation: Irvine: CA, 2007.

10. Trenkle DL, Shankle WR, Azen SP. Detecting cognitive impairment in primary care: performance assessment of three screening instruments. J Alzheimers Dis 2007 11: 323-335.

11. Friston KJ, Holmes AP, Worsley KJ, Poline JP, Frith CD, Frackowiak RSJ. Statistical parametric maps in functional imaging: a general linear approach. Hum Brain Mapp 1995 2: $189-210$.

12. Ashburner J, Friston KJ. Nonlinear spatial normalization using basis functions. Hum Brain Mapp 1999; 7: 254-266.

13. Walther K, Birdsill AC, Glisky EL, Ryan L. Structural brain differences and cognitive functioning related to body mass index in older females. Hum Brain Mapp 2010; 31: 1052-1064

14. Volkow ND, Wang GJ, Telang F, Fowler JS, Goldstein RZ, Alia-Klein N et al. Inverse association between BMI and prefrontal metabolic activity in healthy adults. Obesity (Silver Spring) 2009; 17: 60-65.

15. Laurson KR, Eisenmann JC. Prevalence of overweight among high school football linemen. JAMA 2007; 297: 363-364.

16. Miller EK. The prefrontal cortex and cognitive control. Nat Rev Neurosci 2000; 1: 59-65.
17. Miller EK, Cohen JD. An integrative theory of prefrontal cortex function. Annu Rev Neurosci 2001; 24: 167-202.

18. Guskiewicz KM, Marshall SW, Bailes J, McCrea M, Cantu RC, Randolph C et al. Association between recurrent concussion and late-life cognitive impairment in retired professional football players. Neurosurgery 2005; 57: 719-726.

19. Omalu B, Bailes J, Hamilton RL, Kamboh Ml, Hammers J, Case $\mathrm{M}$ et al. Emerging histomorphologic phenotypes of chronic traumatic encephalopathy [CTE] in American athletes. Neurosurgery 2011; 69: 173-183.

20. Omalu BI, Bailes J, Hammers JL, Fitzsimmons RP. Chronic traumatic encephalopathy, suicides and parasuicides in professional American athletes: the role of the forensic pathologist. Am J Forensic Med Pathol 2010; 31: 130-132.

21. Omalu Bl, Hamilton RL, Kamboh Ml, DeKosky ST, Bailes J. Chronic traumatic encephalopathy (CTE) in a National Football League Player: case report and emerging medicolegal practice questions. J Forensic Nurs 2010; 6: 40-46.

22. De Beaumont L, Brisson B, Lassonde $M$, Jolicoeur $P$. Long-term electrophysiological changes in athletes with a history of multiple concussions. Brain Inj 2007; 21 631-644.

23. Anstey KJ, Cherbuin N, Budge M, Young J. Body mass index in midlife and late-life as a risk factor for dementia: a meta-analysis of prospective studies. Obes Rev 2011; 12 e426-e437.

24. Raji CA, Ho AJ, Parikshak NN, Becker JT, Lopez OL, Kuller LH et al. Brain structure and obesity. Hum Brain Mapp 2010; 31: 353-364.

25. Boeka AG, Lokken KL. Neuropsychological performance of a clinical sample of extremely obese individuals. Arch Clin Neuropsychol 2008; 23: 467-474.

26. Gazdzinski S, Kornak J, Weiner MW, Meyerhoff DJ. Body mass index and magnetic resonance markers of brain integrity in adults. Ann Neurol 2008; 63: 652-657.

27. Tucker AM, Vogel RA, Lincoln AE, Dunn RE, Ahrensfield DC, Allen TW et al. Prevalence of cardiovascular disease risk factors among National Football League players. JAMA 2009; 301: 2111-2119.

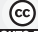

Translational Psychiatry is an open-access journal

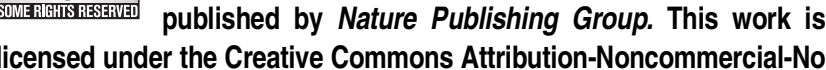
Derivative Works 3.0 Unported License. To view a copy of this license, visit http://creativecommons.org/licenses/by-nc-nd/3.0/ 